\title{
Advances in Rotation-Invariant Texture Analysis
}

\author{
Alfonso Estudillo-Romero and Boris Escalante-Ramirez \\ Universidad Nacional Autonoma de Mexico, Fac. de Ingenieria, Edif. de Posgrado e \\ Investigacion, Ciudad Universitaria, C.P. 04510, Mexico, D.F., Mexico \\ aestudillor@uxmcc2.iimas.unam.mx, boris@servidor.unam.mx
}

\begin{abstract}
Robust rotation invariance has been a matter of great interest in many applications which use low-level features such as textures. In this paper, we propose a method to analyze and capture visual patterns from textures regardless their orientation. In order to achieve rotation invariance, visual texture patterns are locally described as one-dimensional patterns by appropriately steering the Cartesian Hermite coefficients. Experiments with two datasets from the Brodatz album were performed to evaluate orientation invariance. High average precision and recall rates were achieved by the proposed method.
\end{abstract}

Keywords: Texture analysis, steered Hermite transform, image retrieval.

\section{Introduction}

There is no precise definition of texture, but there are intuitive and interesting properties of texture which are generally assumed by researchers. Texture can be described on a spatial neighborhood, whose size and meaning depend upon the texture type and the scale or resolution at which the texture is perceived. We can also relate texture to a set of repetitive patterns, sometimes named texture primitives, taking place into the neighborhood. Texture gray level values can also form a distribution and characterize the texture. The fact that perception of texture has so many dimensions is an important reason why there is no single technique to represent a variety of textures.

Despite the lack of a precise definition, it is often desired that robust texture analysis achieve one of the most important requirements: to be rotation invariant. Early methods realizing the importance of rotation invariance used polarograms [1] and model based methods proposed a circular symmetric autoregressive model [2] and Gaussian Markov random field models (GMRF) 3].

Recent methods based on Wavelet transforms achieve rotation invariance by performing preprocessing stages over the texture image such as polar transformations [4] or a Radon transform [5]. However, one disadvantage of this strategy is the large number of free parameters and consequently a deeper analysis must be done in order to find optimal parameters for each dataset. On the other hand, some methods achieve rotation invariance by performing post-processing stages such as circular shifts over the feature map according to a dominant orientation 6 67] and addition of the different directional coefficients at each scale of analysis [8].

E. Bayro-Corrochano and J.-O. Eklundh (Eds.): CIARP 2009, LNCS 5856, pp. 145-152, 2009.

(C) Springer-Verlag Berlin Heidelberg 2009 
In this work we propose a robust method to extract rotation-invariant features from texture images using the steered Hermite transform. The Hermite transform is known to be in agreement with some processes found in early vision systems. Moreover, the steered Hermite transform proposed in [9] and studied in 10] and 11] provides an efficient way to find meaningful patterns at many orientations and then compress them into a few coefficients. Methods using the Discrete Wavelet Transform (DWT) need extra processing stages before or after the feature extraction process in order to achieve rotation invariance, whereas methods based on Gabor wavelets need filters to be tuned at fixed orientations. The property of coefficient steering, based on the directionality of maximum energy, provides a non-fixed orientation filter design which has the advantage of approximately finding the same features regardless the orientation of the input image.

Section 2 summarizes the Hermite transform theory for two-dimensional signals. In Sect. 3 the steered Hermite transform is presented. The proposed methodology is presented in Sect. 4. Experimental results are reported in Sect. 5 and finally, conclusions and future directions are given in Sect.6.

\section{Hermite Transform}

For the one dimensional case, a polynomial transform $L_{n}(x)$ is a local decomposition technique in which an input signal $L(x)$ is localized through a window $V(x)$ and then expanded into orthogonal polynomials $G_{n}(x)$ at every window position [9]:

$$
L_{n}\left(x_{0}\right)=\int_{x} L(x) G_{n}\left(x_{0}-x\right) V_{n}^{2}\left(x_{0}-x\right) d x .
$$

The Hermite transform arises when $G_{n}$ are the Hermite polynomials $H_{n}(x)$, given by Rodrigues' formula:

$$
H_{n}(x)=(-1)^{n} e^{x^{2}} \frac{d^{n} e^{-x^{2}}}{d x^{n}}, \quad n=0,1,2, \ldots .
$$

and the orthogonal window corresponds to a Gaussian window:

$$
V(x)=\frac{1}{\sqrt{\sqrt{\pi} \sigma}} \cdot e^{-x^{2} / 2 \sigma^{2}} .
$$

Following (1), the expansion coefficients $L_{n}(x)$ can be derived by convolution of the signal $L(x)$ with the Hermite analysis functions $d_{n}(x)$, which are given in terms of the window and Hermite polynomials as:

$$
d_{n}(x)=\frac{(-1)^{n}}{\sqrt{2^{n} n !}} \cdot \frac{1}{\sigma \sqrt{\pi}} H_{n}\left(\frac{x}{\sigma}\right) e^{-x^{2} / \sigma^{2}} .
$$

The Hermite analysis functions can be easily generalized to two dimensions because of the property of being both spatially separable and rotationally symmetric. We then can write the two dimensional analysis functions as:

$$
d_{n-m, m}(x, y)=d_{n-m}(x) d_{m}(y) .
$$


where $n-m$ and $m$ denote the analysis order in $x$ and $y$ direction respectively. As a result, we can expand a given input image $L(x, y)$ into the basis $d_{n-m, m}(x, y)$ as:

$$
L_{n-m, m}\left(x_{0}, y_{0}\right)=\int_{x} \int_{y} L(x, y) d_{n-m, m}\left(x_{0}-x, y_{0}-y\right) d x d y .
$$

for $n=0,1, \ldots, \infty$ and $m=0, \ldots, n$.

\section{Steered Hermite Transform}

A steerable filter is described as a class of filters in which a filter of arbitrary orientation is synthesized as a linear combination of a set of basis filters [12]. Since all Hermite filters are polynomials times a radially symmetric window function, rotated versions of a filter of order $n$ can be constructed by taking linear combinations of the original filters of order $n$. In this way, a more general expression of the original $L_{n-m, m}$ Cartesian Hermite coefficients can be written in terms of the orientation selectivity $\theta$ [11:

$$
L_{n-m, m}^{\theta}\left(x_{0}, y_{0}, \theta\right)=\sum_{k=0}^{n} L_{n-k, k}\left(x_{0}, y_{0}\right) \alpha_{n-k, k}(\theta) .
$$

which has been named the steered Hermite transform in [10]. The terms $\alpha_{n-m, m}(\theta)$ are the Cartesian angular functions of order $n$ which give such orientation selectivity are defined as:

$$
\alpha_{n-m, m}(\theta)=\sqrt{C_{n}^{m}} \cos ^{n-m}(\theta) \sin ^{m}(\theta) .
$$

Considering the ideally rotation of an input image within a circularly symmetric window and assuming that no artifacts are introduced due to interpolation and discretization, we then can assume that there is no lost of information during the rotation process. If this is the case, energy is preserved for each rotated image. Thus, we can write the local energy in terms of the steered Hermite coefficients as:

$$
E_{N}=\sum_{n=0}^{N} \sum_{m=0}^{n}\left[L_{n-m, m}\right]^{2}=\sum_{n=0}^{N} \sum_{m=0}^{n}\left[L_{n-m, m}^{\theta}\right]^{2} .
$$

for all $N \geq 0$. In natural images, many of the image details that are of prime importance, such as edges and lines, can be locally described as one-dimensional patterns, that is, patterns that vary only in one direction (and are constant along the orthogonal direction). One may distinguish 1D local energy terms and 2D local energy terms. Thus, we can split local energy of (9) up to order $N$ as:

$$
E_{N}=\left[L_{0,0}\right]^{2}+E_{N}^{1 D}+E_{N}^{2 D} .
$$

where $L_{0,0}$ represents the DC Hermite coefficient and

$$
E_{N}^{1 D}=\sum_{n=1}^{N}\left[L_{n, 0}^{\theta}\right]^{2}
$$




$$
E_{N}^{2 D}=\sum_{n=1}^{N} \sum_{m=1}^{n}\left[L_{n-m, m}^{\theta}\right]^{2} .
$$

One of the objectives when steering coefficients is to maximize detection of patterns along a given local direction $\theta$. In this way, 10, 11 and 13 propose strategies in which $\theta$ is selected such that $E_{N}^{1 D}$ is maximized. As a consequence, compaction of energy (i.e Hermite coefficients) can be efficiently achieved.

\section{Proposed Methodology}

Figure 1 summarizes the proposed methodology to extract rotation-invariant texture patterns based on the stereed Cartesian Hermite coefficients. Note that this is a general scheme, no classification methods neither distance metrics are involved, leaving this interesting areas open to future investigations. Moreover different texture features are suitable to be extracted from the steered Hermite coefficients.

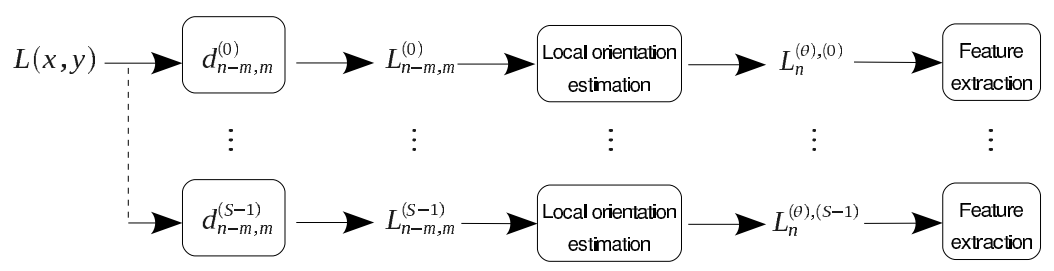

Fig. 1. Texture feature extraction methodology using steered Hermite transform

In this work we used the mean and standard deviation features which seem to better represent on a global sense the behavior of texture patterns distributions for a given frequency (order $n$ ) and scale of analysis. The feature vector is formed by concatenating mean and standard deviation for each steered Hermite coefficient $1 \leq n \leq N$ at every scale of analysis $s$, where $0 \leq s \leq S-1$ and $S$ represents the number of scales:

$$
\boldsymbol{f}=\left[\mu_{1}^{(0)}, \sigma_{1}^{(0)}, \ldots, \mu_{N}^{(0)}, \sigma_{N}^{(0)}, \ldots, \mu_{1}^{(1)}, \sigma_{1}^{(1)}, \ldots, \mu_{N}^{(S-1)}, \sigma_{N}^{(S-1)}\right] .
$$

\section{Experimental Results}

The purpose of the following experiments was to evaluate the ability to extract rotation-invariant visual patterns from texture images with the proposed methodology. Two experiments proposed in early works were reproduced. Although experiments can hold for a particular application such as image retrieval or indexing, our principal contribution is a method to analyze and capture visual patterns regardless their orientation.

Evaluation of texture analysis methods is frequently presented as the behavior of the average of both precision and recall as functions of a requested (query) 
number of images. Let $n_{g}$ be the number of "ground truth" texture images for the class $c$ and let $n_{k}$ be the correct number of retrieved "ground truth" texture images when $k$ queries are performed. Then, precision is defined as:

$$
P_{c}=\frac{n_{k}}{k} \text {. }
$$

and recall as:

$$
R_{c}=\frac{n_{k}}{n_{g}} .
$$

Note that perfect scores precision and recall are obtained when the number of delivered "ground truth" texture images equals the number of queries. By computing the average of both precision and recall for each class and for different $k$ queries it is possible to evaluate the robustness of the rotation-invariant texture analysis method.

The Brodatz texture image dataset [14 was used in both experiments. A Hermite decomposition was performed up to $N=8$ with four scales of analysis. Thus, a feature vector of 64 elements was formed by concatenating mean and standard deviation for each steered Hermite coefficient.

The similarity measure was obtained by computing the distance between the feature vectors using the Canberra distance metric:

$$
d=\sum_{i=1}^{z} \frac{\left|\boldsymbol{f}_{i}-\boldsymbol{g}_{i}\right|}{\left|\boldsymbol{f}_{i}\right|+\left|\boldsymbol{g}_{i}\right|} .
$$

Experiment I. For this experiment, following the configuration of dataset 4 presented in 4], an image dataset of 25 texture classes from the Brodatz album was prepared. First, each $512 \times 512$ texture image was rotated with 36 equally spaced angles, ranging from 0 to $35 \pi / 36$ radians with incremental step size of $\pi / 36$. We do not rotate beyond the upper limit $35 \pi / 36$ radians because it would have redundant rotated texture images. Each rotated texture image is then partitioned from the center of the image to reach $128 \times 128$ pixels. As a result 36 rotated texture images comprise the "ground truth" images for each texture class and a dataset of $25 \times 36$ texture images was formed with rotation of a single area.

Figure 2 shows graphs of the precision and recall average rates. We noted how well the steered Hermite transform performed for the majority of 36 "ground truth" texture images, that is, $99.88 \%$ retrieval performance when $P(\%)=R(\%)$ (i.e. for $k=36$ first retrieved texture images). Comparing our results with the presented in 4, for dataset with rotation only from a single area, we obtained similar results $(\mathrm{P}=100 \%)$ even for all the first $k=32$ retrieved texture images.

Experiment II. For this experiment dataset of experiment I was extended to 108 Brodatz texture images. First, each $512 \times 512$ texture image was rotated with 16 equally spaced angles, ranging from 0 to $15 \pi / 16$ radians with incremental step size of $\pi / 16$ as proposed in [8]. Each rotated texture image is then partitioned 

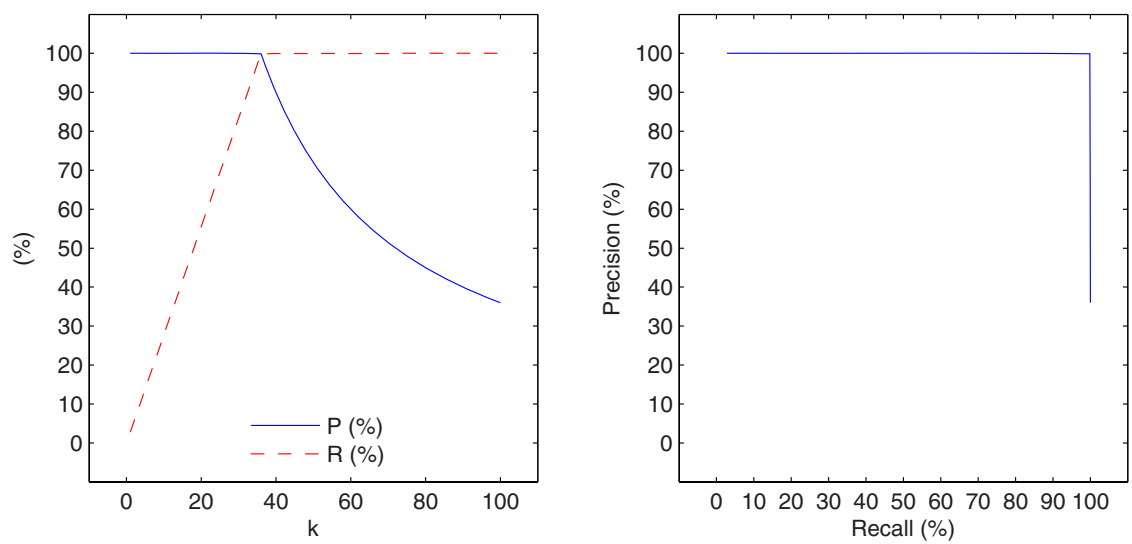

Fig. 2. Average retrieval performance. Note that approximately a perfect average compromise between precision and recall $(99.88 \%)$ was obtained when retrieving the first 36 "ground truth" texture images.
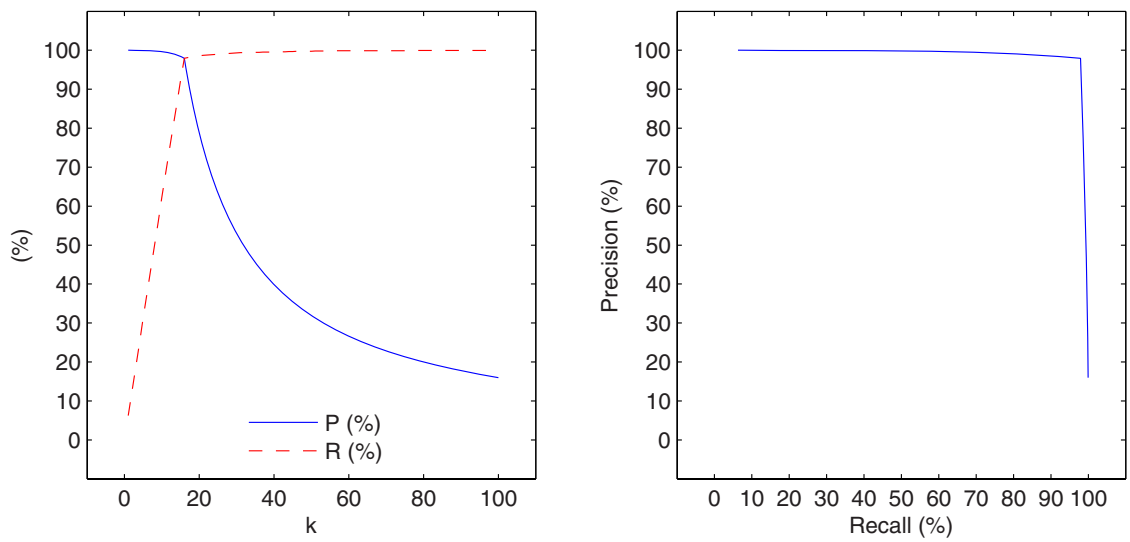

Fig. 3. Average retrieval performance of experiment II. Note that approximately a perfect average compromise between precision and recall (97.92\%) was obtained when retrieving the first 16 "ground truth" texture images.

from the center of the image to reach $128 \times 128$ pixels. As a result 16 rotated texture images comprise the "ground truth" images for each texture class and a dataset of $108 \times 16$ texture images was formed with rotation of a single area.

Figure 3 shows graphs of the precision and recall average rates. The steered Hermite transform-based features got performance retrieval of $97.93 \%$ when $P(\%)=R(\%)$ (i.e. for $k=16$ first retrieved texture images). In $[8$ and 6 ] experiments were performed with similar texture images datasets. Han and Ma 8 propose a Gabor-based rotation-invariant method and compare it with previous Gabor-based methods. In [6] a rotation-invariant method using waveletbased hidden Markov trees was proposed and reported an average retrieval rate 
of $91.25 \%$. Comparing results, it seems that the proposed methodology outperformed average retrieval rates of the methods above mentioned. We noticed in our experiment that for all the first $k=3$ retrieved texture images the average precision rate was $\mathrm{P}=100 \%$.

\section{Conclusions}

In this work, a rotation-invariant texture analysis methodology was proposed. Texture analysis was performed taking into consideration visual information from the texture images. First, the analysis functions of the Hermite transform performed filtering and extracted visual details which were then locally described as one-dimensional patterns by appropriately steering the Cartesian Hermite coefficients. Mean and standard deviation were computed from each steered Hermite coefficient and concatenated to form a vector of 64 features.

Results showed that important visual patterns are well extracted after the steering of Cartesian Hermite coefficients regardless their orientation. Moreover, we observed an important comprise between average precision and recall rates with the present method outperforming results previously reported with other methods.

Although evaluation of the proposed method was conducted for texture image retrieval, our principal contribution is a method to analyze and capture visual patterns regardless their orientation. Therefore, many applications that use texture features can be implemented following the proposed scheme. Future works will include classification and segmentation evaluations.

\section{Acknowledgements}

This work was supported by UNAM-PAPIIT IN106608.

\section{References}

1. Davis, L.S.: Polarograms: A new tool for image texture analysis. Pattern Recognition 13(3), 219-223 (1981)

2. Kashyap, R.L., Khotanzad, A.: A model-based method for rotation invariant texture classification. IEEE Trans. PAMI 8(4), 472-481 (1986)

3. Cohen, F., Fan, Z., Patel, M.: Classification of rotated and scaled textured images using gaussian markov random field models. IEEE Trans. PAMI 13(2), 192-202 (1991)

4. Pun, C.M., Lee, M.C.: Log-polar wavelet energy signatures for rotation and scale invariant texture classification. IEEE Trans. PAMI 25(5), 590-603 (2003)

5. Jafari-Khouzani, K., Soltanian-Zadeh, H.: Rotation-invariant multiresolution texture analysis using radon and wavelet transforms. IEEE Transactions on Image Processing 14(6), 783-795 (2005)

6. Rallabandi, V.R., Rallabandi, V.S.: Rotation-invariant texture retrieval using wavelet-based hidden markov trees. Signal Processing 88(10), 2593-2598 (2008) 
7. Montoya-Zegarra, J.A., Papa, J.P., Leite, N.J., da Silva Torres, R., Falco, A.X.: Learning how to extract rotation-invariant and scale-invariant features from texture images. EURASIP Journal on Advances in Signal Processing 691924 (2008)

8. Han, J., Ma, K.K.: Rotation-invariant and scale-invariant gabor features for texture image retrieval. Image and Vision Computing 25(9), 1474-1481 (2007)

9. Martens, J.B.: The hermite transform-theory. IEEE Transactions on Acoustics, Speech and Signal Processing 38(9), 1595-1606 (1990)

10. van Dijk, A.M., Martens, J.B.: Image representation and compression with steered hermite transforms. Signal Processing 56(1), 1-16 (1997)

11. Silvan-Cardenas, J., Escalante-Ramirez, B.: The multiscale hermite transform for local orientation analysis. IEEE Transactions on Image Processing 15(5), 1236 $1253(2006)$

12. Freeman, W., Adelson, E.: The design and use of steerable filters. IEEE Trans. PAMI 13(9), 891-906 (1991)

13. Martens, J.B.: The Hermite transform: a survey. EURASIP Journal of Applied Signal Processing 26145 (2006)

14. Brodatz, P.: Texture: a photographic album for artists and designers. Dover, New York (1966) 